\title{
Ordered Variables and Their Concomitants under Extropy via COVID-19 Data Application
}

\author{
Mohamed S. Mohamed (D), Alanazi Talal Abdulrahman (iD, ${ }^{2}$ Zahra Almaspoor (D), \\ and M. Yusuf ${ }^{4}$ \\ ${ }^{1}$ Department of Mathematics, Faculty of Education, Ain Shams University, Cairo 11341, Egypt \\ ${ }^{2}$ Department of Mathematics, Faculty of Science, Ha'il University, Hail, Saudi Arabia \\ ${ }^{3}$ Department of Statistics, Yazd University, P.O. Box 89175-741, Yazd, Iran \\ ${ }^{4}$ Department of Mathematics, Faculty of Science, Helwan University, Cairo, Egypt
}

Correspondence should be addressed to Zahra Almaspoor; zahra.ferry21@gmail.com

Received 19 April 2021; Accepted 21 June 2021; Published 6 July 2021

Academic Editor: Ning Cai

Copyright (C) 2021 Mohamed S. Mohamed et al. This is an open access article distributed under the Creative Commons Attribution License, which permits unrestricted use, distribution, and reproduction in any medium, provided the original work is properly cited.

\begin{abstract}
Extropy, as a complementary dual of entropy, has been discussed in many works of literature, where it is declared for other measures as an extension of extropy. In this article, we obtain the extropy of generalized order statistics via its dual and give some examples from well-known distributions. Furthermore, we study the residual and past extropy for such models. On the other hand, based on Farlie-Gumbel-Morgenstern distribution, we consider the residual extropy of concomitants of $m$-generalized order statistics and present this measure with some additional features. In addition, we provide the upper bound and stochastic orders of it. Finally, nonparametric estimation of the residual extropy of concomitants of $m$-generalized order statistics is included using simulated and real data connected with COVID-19 virus.
\end{abstract}

\section{Introduction}

Shannon [1] introduced a well-known vintage measure of uncertainty called Shannon entropy. This information theoretic entropy manipulates in diverse fields such as financial analysis, computer science, and medical research. The extropy proposed by Lad et al. [2] is an accomplishment to notions of information based on entropy. They exhibited that entropy has a complementary dual function known as "extropy." In the view of extropy in discrete density, the extropy measure $-\sum_{i=1}^{N}\left(1-\theta_{i}\right) \log \left(1-\theta_{i}\right)$ is neatly closer to $(-1 / 2) \sum_{i=1}^{N} \theta_{i}^{2}$ when the range of possibilities increases (as a result of larger $N$ ). Therefore, to realize extropy for a continuous density, the extropy of a nonnegative continuous random variable (r.v.) $X$, with probability density function (PDF) $f(x)$ is defined as

$$
J(X)=\frac{-1}{2} \int_{0}^{\infty} f^{2}(x) \mathrm{d} x .
$$

The extropy measure has been developed for ordered variables. Qiu [3] was the first to apply extropy for order statistics and record values and present several of their properties. After that, the researchers manifested to present extension measures of extropy. Qiu and Jia [4] investigated the connotation of residual extropy of a nonnegative r.v. as

$$
J^{t}(X)=J^{R}(X ; t)=\frac{-1}{2 \bar{F}^{2}(t)} \int_{t}^{\infty} f^{2}(x) \mathrm{d} x, \quad t \geq 0 .
$$

Qiu et al. [5] presented a mixed systems lifetime via extropy and obtained some features and bounds of it. Recently, Jose and Sathar $[6,7]$ exploited the residual and past extropy of $k$-records, respectively, emerging from any continuous distribution. For extra studies on extropy, see Qiu and Jia [8], Yang et al. [9], Noughabi and Jarrahiferiz [10], Raqab and Qiu [11], and Lad et al. [12].

Krishnan et al. [13] presented the past extropy, for a fixed $t>0$, for past lifetime of r.v. $X_{t}=[t-X \mid X \leq t]$ as follows: 


$$
J_{t}(X)=J_{P}(X ; t)=\frac{-1}{2 F^{2}(t)} \int_{0}^{t} f^{2}(x) \mathrm{d} x
$$

Jahanshahi et al. [14] proposed cumulative residual extropy (CREX). For a nonnegative r.v. $X$ with an absolutely continuous survival function $\bar{F}$, the CREX is given by

$$
\zeta(X)=\frac{-1}{2} \int_{0}^{\infty} \bar{F}^{2}(x) \mathrm{d} x
$$

In analogy with Jahanshahi et al. [14], Abdul Sathar and Dhanya [15] introduced the CREX and refer to it as survival extropy. Moreover, they conduct the dynamic survival extropy as

$$
\zeta(X ; t)=\frac{-1}{2 \bar{F}^{2}(t)} \int_{t}^{\infty} \bar{F}^{2}(x) \mathrm{d} x, \quad t \geq 0 .
$$

It is easy to observe that extropy and its related measures are constantly negative.

The connotation of generalized order statistics (gos) that contains all forms of ordered random observations was first proposed by Kamps [16]. Let $k \geq 1, n \in \mathbb{N}$, $\widetilde{m}=\left(m_{1}, \ldots, m_{n-1}\right) \in \mathbb{R}^{n-1}$ be parameters such that $M_{r}=\sum_{j=r}^{n-1} m_{j}, \gamma_{r}=k+n-r+M_{r} \geq 1$ for all $1 \leq r \leq n-1$. For a subclass of gos (called $m$-gos), where $m_{1}=m_{2}=\cdots=m_{n-1}=m$, the PDF of the $r$ th $m-$ gos, $X_{(r ; n, k, m)}$, can be written as

$$
f_{(r ; n, k, m)}(x)=\frac{c_{r-1}}{(r-1) !}(1-F(x))^{\gamma_{r}-1} f(x) g_{m}^{r-1}(F(x)),
$$

where $c_{r-1}=\prod_{j=1}^{r} \gamma_{j}, \gamma_{r}=k+(n-r)(m+1)$, for $0<z<1$,

$$
g_{m}(z)= \begin{cases}\frac{1-(1-z)^{m+1}}{m+1}, & m \neq-1, \\ -\ln (1-z), & m=-1 .\end{cases}
$$

We will write $g_{m}(z)=\left(1-(1-z)^{m+1}\right) /(m+1)$, $0<z<1$ and for all $m$, since $\lim _{m \longrightarrow-1}\left(1-(1-z)^{m+1}\right) /(m+1)=-\ln (1-z)$.

Based on descending ordered r.v.'s, Pawlas and Szynal [17] and Burkschat et al. [18] presented the dual generalized order statistics (dgos). By the same manner and parameters in $m-$ gos, when $m_{1}=m_{2}=\cdots=m_{n-1}=m$, the PDF of $m-$ $\operatorname{dgos} X_{d(r ; n, k, m)}$ is defined by

$$
f_{d(r ; n, k, m)}(x)=\frac{c_{r-1}}{(r-1) !}\left(\frac{1}{m+1}\right)^{r-1}(F(x))^{\gamma_{r}-1}\left(1-F^{m+1}(x)\right)^{r-1} f(x) .
$$

The concept of concomitants of ordinary order statistic was derived by David et al. [19]. Let $\left(X_{i}, Y_{i}\right), i=1,2, \ldots, n$, be $n$ pairs of independent r.v.'s drawn from some bivariate distributions with cumulative distribution function (CDF) $F(x, y)$. Let $X_{(r ; n)}$ be the $r$ th order statistic, then the r.v. $Y$ concerned with $X_{(r ; n)}$ is called the concomitant of $r$ th order statistics and is specified by $Y_{[r ; n]}$.
The Farlie-Gumbel-Morgenstern (FGM) family is an extremely supple class of bivariate family; it was primarily derived by Morgenstern [20], which is set by CDF and PDF, respectively, as follows:

$$
\begin{aligned}
& \quad F_{X, Y}(x, y) \\
& \quad=F_{X}(x) F_{Y}(y)\left[1+\alpha\left(1-F_{X}(x)\right)\left(1-F_{Y}(y)\right)\right], \\
& f_{X, Y}(x, y) \\
& \quad=f_{X}(x) f_{Y}(y)\left[1+\alpha\left(2 F_{X}(x)-1\right)\left(2 F_{Y}(y)-1\right)\right],
\end{aligned}
$$

where $F_{X}(x), F_{Y}(y)$ and $f_{X}(x), f_{Y}(y)$ are the marginal CDF's and PDF's of $X$ and $Y$, respectively, $-1 \leq \alpha \leq 1$. If the dependent parameter $\alpha=0$, then $X$ and $Y$ are not dependent. Beg and Ahsanullah [21] introduced the PDF of the concomitant of $m-\operatorname{gos} Y_{[r ; n, k, m]}, 1 \leq r \leq n$, under the FGM family as follows:

$$
g_{[r ; n, k, m]}(y)=f_{Y}(y)\left[1+\alpha T^{*}(r ; n, k, m)\left(2 F_{Y}(y)-1\right)\right],
$$

where

$$
T^{*}(r ; n, k, m)=1-2 \frac{\prod_{j=1}^{r} \gamma_{j}}{\prod_{i=1}^{r}\left(\gamma_{i}+1\right),}
$$

with parameters $n \in \mathbb{N}, \quad k \geq 1, \quad m \in \mathbb{R}$, such that $\gamma_{r}=k+(n-r)(m+1) \geq 1$, for all $1 \leq r \leq n$.

Throughout this paper, we propose the extropy of $m-$ gos and $m$-dgos and study those models for the related measures of extropy. In the second part of the paper, we deal with the concomitants of $m$-gos of FGM family to extract the residual extropy and give some of its properties. The paper is organized as follows: Section 2 contains extropy of $m$ - gos and $m$-dgos obtained from uniform distribution. Moreover, we obtain them for any distribution in terms of the obtained extropy from uniform distribution. Meanwhile, we produce some examples of some well-known distributions. Furthermore, we obtain the lower bound of the extropy of $m$ - gos in terms of the mode. In addition, the residual and past extropy of $m-$ gos and $m-$ dgos is considered in Section 3. In Section 4, we derive the residual extropy of concomitants of $m$-gos of FGM family and discuss its relation with the stop-loss transform and Gini index. Besides, we consider this model in terms of its upper bound and produce some examples on it. Finally, in Section 5, real-life data connected with the COVID-19 virus is applied for the nonparametric estimation of residual extropy of concomitants of order statistics under the FGM family.

\section{Extropy of $m$-Generalized Order Statistics and Its Dual}

In this section, we discuss the extropy of $m$ - gos and $m-$ dgos for uniform distribution and for any distribution, which depends on beta function and its generalized first kind. 
Based on uniform distribution $U(0,1)$, the extropy of the $r$ th $m$ - gos, $X_{(r ; n, k, m)}, 1 \leq r \leq n$, can be obtained by the following theorem.

Theorem 1. Suppose the nonnegative continuous r.v. $X$ be emerging from $U(0,1)$ distribution. Let $U_{(r ; n, k, m)}$ be its rth $m$ - gos; then, from (1) and (6), the extropy of $U_{(r ; n, k, m)}$ is given by

$$
\begin{aligned}
J\left(U_{(r ; n, k, m)}\right) & =\frac{-\left(c_{r-1}\right)^{2}}{2((r-1) !)^{2}}\left(\frac{1}{m+1}\right)^{2 r-1} B\left(2 r-1, \frac{2 \gamma_{r}-1}{m+1}\right) \\
& =\frac{-\left(c_{r-1}\right)^{2}}{2((r-1) !)^{2}} \frac{(2 r-2) !}{\left(\prod_{i=1}^{r}\left(\gamma_{1}+\gamma_{i}-1\right)\right)\left(\prod_{i=1}^{r-1}\left(\gamma_{r}+\gamma_{1+i}-1\right)\right) .}
\end{aligned}
$$

Proof. From (1) and (6), we have

$$
\begin{aligned}
J\left(U_{(r ; n, k, m)}\right) & =\frac{-1}{2} \int_{0}^{1} f_{(r ; n, k, m)}^{2}(u) \mathrm{d} u \\
& =A_{(r ; n, k, m)} \int_{0}^{1}(1-u)^{2 \gamma_{r}-2}\left(1-(1-u)^{m+1}\right)^{2 r-2} \mathrm{~d} u
\end{aligned}
$$

where $\quad A_{(r ; n, k, m)}=(-1 / 2)\left(\left(c_{r-1}\right)^{2} /((r-1) !)^{2}\right)$ $1))^{2 r-2}$. Using the transformation $z=1-u$, thus

$$
\begin{aligned}
J\left(U_{(r ; n, k, m)}\right) & =A_{(r ; n, k, m)} \int_{0}^{1} z^{2 \gamma_{r}-2}\left(1-z^{m+1}\right)^{2 r-2} \mathrm{~d} z \\
& =A_{(r ; n, k, m)} \frac{1}{m+1} B\left(2 r-1, \frac{2 \gamma_{r}-1}{m+1}\right),
\end{aligned}
$$

where $\quad B(x, y / z)=z \int_{0}^{1} t^{y-1}\left(1-t^{z}\right)^{x-1} \mathrm{~d} t=(\Gamma(x) \Gamma(y / z) /$ $\Gamma(x+(y / z)))$ is the beta function. Therefore, we can reduce the beta function in (15) as follows:

$$
\begin{aligned}
B\left(2 r-1, \frac{\left(2 \gamma_{r}-1\right)}{(m+1)}\right)= & \frac{\Gamma(2 r-1) \Gamma\left(\left(2 \gamma_{r}-1\right) /(m+1)\right)}{\Gamma\left(2 r-1+\left(\left(2 \gamma_{r}-1\right) /(m+1)\right)\right)} \\
= & \frac{(2 r-2) ! \Gamma\left(\left(2 \gamma_{r}-1\right) /(m+1)\right)}{\underbrace{\left(2 r+\left(\left(2 \gamma_{r}-1\right) /(m+1)\right)-2\right)\left(2 r+\left(\left(2 \gamma_{r}-1\right) /(m+1)\right)-3\right), \ldots,\left(\left(\left(2 \gamma_{r}-1\right) /(m+1)\right)+r-1\right)}_{\mathbf{r}-\text { times }}} \\
& \times \underbrace{\frac{\left(\left(\left(2 \gamma_{r}-1\right) /(m+1)\right)+r-2\right)\left(\left(\left(2 \gamma_{r}-1\right) /(m+1)\right)+r-3\right), \ldots,\left(\left(2 \gamma_{r}-1\right) /(m+1)\right) \Gamma\left(\left(2 \gamma_{r}-1\right) /(m+1)\right)}{1}}_{(\mathbf{r}-1) \text {-times }}
\end{aligned}
$$

Furthermore,

$$
\begin{aligned}
\left(\frac{1}{(m+1)}\right)^{2 r-1} B\left(2 r-1, \frac{\left(2 \gamma_{r}-1\right)}{(m+1)}\right) & =\left(\frac{1}{(m+1)}\right)^{r}\left(\frac{1}{(m+1)}\right)^{r-1} B\left(2 r-1, \frac{\left(2 \gamma_{r}-1\right)}{(m+1)}\right) \\
& =\left(\frac{1}{(m+1)}\right)^{r}\left(\frac{1}{(m+1)}\right)^{r-1} \underbrace{\frac{\left.\left(2 r+\left(\left(2 \gamma_{r}-1\right) /(m+1)\right)-2\right), \ldots,\left(\left(2 \gamma_{r}-1\right) /(m+1)\right)+r-1\right)}{1}}_{\text {r-times }} \\
& \times \frac{\left.1\left(\left(2 \gamma_{r}-1\right) /(m+1)\right)+r-2\right)\left(\left(\left(2 \gamma_{r}-1\right) /(m+1)\right)+r-3\right), \ldots,\left(\left(2 \gamma_{r}-1\right) /(m+1)\right)}{(\mathbf{r}-1)-\text { times }} \\
= & \frac{(2 r-2) !}{\left(\prod_{i=1}^{r}\left(\gamma_{1}+\gamma_{i}-1\right)\left(\prod_{i=1}^{r-1}\left(\gamma_{r}+\gamma_{1+i}-1\right)\right),\right.}
\end{aligned}
$$

and the result is taken after.

In the following theorem, we derive the extropy of $m$-gos, $X_{(r ; n, k, m)}$, from any continuous distribution based on extropy of $m$ - gos emerging from $U(0,1)$.
Theorem 2. Let $X$ be a continuous r.v. that is nonnegative with $C D F F(x)$. Then, from (1), (6), and (13), the extropy of the rth $m$ - gos, $X_{(r ; n, k, m)}, 1 \leq r \leq n$, is given by

$$
J\left(X_{(r ; n, k, m)}\right)=J\left(U_{(r ; n, k, m)}\right) \mathbb{E}\left(f\left(F^{-1}\left(1-V_{r}\right)\right)\right),
$$


where $V_{r}$ has generalized beta of first kind distribution (i.e., $\left.V_{r} \sim G B 1\left(x ; m+1,\left(2 \gamma_{r}-1\right) /(m+1), 2 r-1\right)\right) \quad$ and Proof. From (1) and (6), we have GB1 $(x ; a, p, q)$ has PDF:

$$
f(x ; a, p, q)=\frac{a x^{a p-1}\left(1-x^{a}\right)^{q-1}}{B(p, q)}, \quad 0<x<1, a, p, q>0 .
$$

$$
J\left(X_{(r ; n, k, m)}\right)=\frac{-1}{2} \int_{0}^{\infty} f_{(r ; n, k, m)}^{2}(x) \mathrm{d} x=A_{(r ; n, k, m)} \int_{0}^{\infty}(1-F(x))^{2 \gamma_{r}-2}\left(1-(1-F(x))^{m+1}\right)^{2 r-2} f^{2}(x) \mathrm{d} x
$$

Putting $u=1-F(x)$, thus

$$
\begin{aligned}
J\left(X_{(r ; n, k, m)}\right)= & A_{(r ; n, k, m)} \int_{0}^{1} u^{2 \gamma_{r}-2}\left(1-u^{m+1}\right)^{2 r-2} f\left(F^{-1}(1-u)\right) \mathrm{d} u \\
= & A_{(r ; n, k, m)} \frac{1}{m+1} B\left(2 r-1,\left(2 \gamma_{r}-1\right) /(m+1)\right) \\
& \times \frac{m+1}{B\left(2 r-1,\left(2 \gamma_{r}-1\right) /(m+1)\right)} \int_{0}^{1} u^{2 \gamma_{r}-2}\left(1-u^{m+1}\right)^{2 r-2} f\left(F^{-1}(1-u)\right) \mathrm{d} u \\
= & J\left(U_{(r ; n, k, m)}\right) \mathbb{E}\left(f\left(F^{-1}\left(1-V_{r}\right)\right)\right),
\end{aligned}
$$

which proves the theorem.

From the previous theorem, we show that the extropy of $m$ - gos is the product of extropy of $m$ - gos emerging from $U(0,1)$ distribution and expectation of the first kind generalized beta distributed r.v.

Now, we will give some special cases on Theorem 2 by the following examples.

Example 1. Suppose the nonnegative continuous r.v. $X$ arising from exponential distribution, denoted by $\operatorname{EXP}(\lambda)$, with $\operatorname{CDF}$

$$
\begin{aligned}
F(x) & =1-e^{-\lambda x}, \quad \lambda>0, x>0, \\
F^{-1}(x) & =\frac{-1}{\lambda} \ln (1-x) .
\end{aligned}
$$

Therefore,

$$
f\left(F^{-1}(1-u)\right)=\lambda u
$$

Thus,

$\mathbb{E}\left(f\left(F^{-1}\left(1-V_{r}\right)\right)\right)=\frac{\lambda}{B\left(2 r-1,\left(2 \gamma_{r}-1\right) /(m+1)\right)} B\left(2 r-1, \frac{2 \gamma_{r}}{m+1}\right)$.

Thence,

$$
J\left(X_{(r ; n, k, m)}\right)=\frac{-\lambda\left(c_{r-1}\right)^{2}}{2((r-1) !)^{2}}\left(\frac{1}{m+1}\right)^{2 r-1} B\left(2 r-1, \frac{2 \gamma_{r}}{m+1}\right) .
$$

By the same manner in (17), we can reduce (25) as

$$
J\left(X_{(r ; n, k, m)}\right)=\frac{-\lambda\left(c_{r-1}\right)^{2}}{2((r-1) !)^{2}} \frac{(2 r-2) !}{\left(\prod_{i=1}^{r}\left(\gamma_{1}+\gamma_{i}\right)\right)\left(\prod_{i=1}^{r-1}\left(\gamma_{r}+\gamma_{1+i}\right)\right) .}
$$

Example 2. Suppose the nonnegative continuous r.v. $X$ arising from Pareto distribution with $\mathrm{CDF}$

$$
\begin{array}{r}
F(x)=1-\left(\frac{x}{\sigma}\right)^{-\alpha}, \quad \sigma>0, x>\sigma, \\
F^{-1}(x)=\sigma(1-x)^{(-1 / \alpha)},
\end{array}
$$

Therefore,

$$
f\left(F^{-1}(1-u)\right)=\frac{\alpha}{\sigma} u^{1+(1 / \alpha)} .
$$

Thus,

$$
\mathbb{E}\left(f\left(F^{-1}\left(1-V_{r}\right)\right)\right)=\frac{\alpha}{\sigma B\left(2 r-1,\left(2 \gamma_{r}-1\right) /(m+1)\right)} B\left(2 r-1, \frac{2 \gamma_{r}+(1 / \alpha)}{m+1}\right) .
$$

Thence,

$$
J\left(X_{(r ; n, k, m)}\right)=\frac{-\alpha\left(c_{r-1}\right)^{2}}{2 \sigma((r-1) !)^{2}}\left(\frac{1}{m+1}\right)^{2 r-1} B\left(2 r-1, \frac{2 \gamma_{r}+(1 / \alpha)}{m+1}\right) .
$$

In the next corollary, the lower bound for the extropy of $m$ - gos will be obtained in terms of the extropy of $m$ - gos emerging from $U(0,1)$ and the mode of the distribution. 
Corollary 1. Suppose that $M$ is the mode of the r.v. X such that $M=f(m)$ which exists; therefore,

$$
J\left(X_{(r ; n, k, m)}\right) \geq J\left(U_{(r ; n, k, m)}\right) M .
$$

2.1. Extropy of Dual m-Generalized Order Statistics. Based on $U(0,1)$ distribution, from (1) and (8) and Theorem 1, we can obviously see that the extropy of $r$ th $m-$ gos, $U_{(r ; n, k, m)}$, is the same as the extropy of the $r$ th $m-\operatorname{dgos} U_{d(r ; n, k, m)}$. On the other hand, we can obtain the extropy of $r$ th $m$-dgos, $X_{d(r ; n, k, m)}, 1 \leq r \leq n$, for any distribution from the following theorem.
Theorem 3. Let $X$ be a continuous r.v. that is nonnegative with CDF F ( $x$ ) distribution. Then, from (1), (8), and (13), the extropy of the rth $m-d g o s, X_{d(r ; n, k, m)}, 1 \leq r \leq n$, is given by

$$
J\left(X_{d(r ; n, k, m)}\right)=J\left(U_{d(r ; n, k, m)}\right) \mathbb{E}\left(f\left(F^{-1}\left(V_{r}\right)\right)\right),
$$

where $V_{r} \sim G B 1\left(x ; m+1,\left(2 \gamma_{r}-1\right) /(m+1), 2 r-1\right)$ with $P D F$ defined in (19).

Proof. From (1) and (8), we have

$$
\begin{aligned}
J\left(X_{d(r ; n, k, m)}\right) & =\frac{-1}{2} \int_{0}^{\infty} f_{d(r ; n, k, m)}^{2}(x) \mathrm{d} x \\
& =A_{(r ; n, k, m)} \int_{0}^{\infty}(F(x))^{2 \gamma_{r}-2}\left(1-(F(x))^{m+1}\right)^{2 r-2} f^{2}(x) \mathrm{d} x
\end{aligned}
$$

Putting $u=F(x)$, thus

$$
\begin{aligned}
J\left(X_{d(r ; n, k, m)}\right)= & A_{(r ; n, k, m)} \frac{1}{m+1} B\left(2 r-1, \frac{\left(2 \gamma_{r}-1\right)}{(m+1)}\right) \\
& \times \frac{m+1}{B\left(2 r-1,\left(2 \gamma_{r}-1\right) /(m+1)\right)} \int_{0}^{1} u^{2 \gamma_{r}-2}\left(1-u^{m+1}\right)^{2 r-2} f\left(F^{-1}(u)\right) \mathrm{d} u \\
= & J\left(U_{d(r ; n, k, m)}\right) \mathbb{E}\left(f\left(F^{-1}\left(V_{r}\right)\right)\right),
\end{aligned}
$$

where the extropy of $r$ th $m-\operatorname{dgos} J\left(U_{d(r ; n, k, m)}\right)=J$ $\left(U_{(r ; n, k, m)}\right)$ obtained in (13), which proves the theorem.

\section{Residual and Past Extropy of $\boldsymbol{m}$-Generalized Order Statistics and Its Dual}

Under the condition of parameters in (6), Kamps [16] obtained the CDF of $m$-gos, $X_{(r ; n, k, m)}, 1 \leq r \leq n$, as follows:

$$
F_{(r ; n, k, m)}(x)=1-c_{r-1}(1-F(x))^{\gamma_{r}} \sum_{j=0}^{r-1} \frac{1}{j ! c_{r-j-1}}\left[\frac{1}{m+1}\left(1-(1-F(x))^{m+1}\right)\right]^{j}
$$

where $c_{r-j-1}=\prod_{i=1}^{r-j} \gamma_{i}$. Furthermore, Shahbaz et al. [22] derived the CDF of both $m$-gos and $m$ - dgos, $X_{(r ; n, k, m)}$, $X_{d(r ; n, k, m)}, 1 \leq r \leq n$, respectively, in terms of incomplete beta function ratio as follows:

$$
\begin{gathered}
F_{(r ; n, k, m)}(x)=I_{\beta[F(x)]}\left(r, \frac{\gamma_{r}}{m+1}\right), \\
F_{d(r ; n, k, m)}(x)=I_{\beta^{*}[F(x)]}\left(r, \frac{\gamma_{r}}{m+1}\right),
\end{gathered}
$$

where $I_{x}(a, b)=\int_{0}^{x} t^{a-1}(1-t)^{b-1} \mathrm{~d} t$ is incomplete beta function ratio, $\beta[F(x)]=1-(1-F(x))^{m+1}, \quad \beta^{*}[F(x)]$ $=F(x)^{m+1}$.

In the next theorems, we obtain the residual and past extropy of $m$ - gos and $m$-dgos emerging from any distribution.

Theorem 4. Let $X$ be a continuous r.v. that is nonnegative with CDF F $(x)$ distribution. Then, from (2), (6), and (36), the residual extropy of rth $m-$ gos, $X_{(r ; n, k, m)}, 1 \leq r \leq n$, is given by

$$
J\left(X_{(r ; n, k, m)}\right)=\frac{1}{\bar{F}_{(r ; n, k, m)}^{2}(x)} J\left(U_{(r ; n, k, m)}\right) \mathbb{E}\left(f\left(F^{-1}\left(1-V_{r}\right)\right) \mid V_{r}<1-F(t)\right),
$$


where $V_{r} \sim G B 1\left(x ; m+1,\left(2 \gamma_{r}-1\right) /(m+1), 2 r-1\right)$ with PDF defined in (19) and $\bar{F}_{(r ; n, k, m)}(x)=1-F_{(r ; n, k, m)}(x)$ defined in (36).
Theorem 5. Let $X$ be a continuous r.v. that is nonnegative with CDF F ( $x$ ) distribution. Then, from (3), (6), and (36), the past extropy of the rth $m-$ gos, $X_{(r ; n, k, m)}, 1 \leq r \leq n$, is given by

$$
J\left(X_{(r ; n, k, m)}\right)=\frac{1}{F_{(r ; n, k, m)}^{2}(x)} J\left(U_{(r ; n, k, m)}\right) \mathbb{E}\left(f\left(F^{-1}\left(1-V_{r}\right)\right) \mid V_{r}>1-F(t)\right),
$$

where $V_{r} \sim G B 1\left(x ; m+1,\left(2 \gamma_{r}-1\right) /(m+1), 2 r-1\right)$ with PDF defined in (19) and $F_{(r ; n, k, m)}(x)$ defined in (36).
Theorem 6. Let $X$ be a continuous r.v. that is nonnegative with CDF F ( $x$ ) distribution. Then, from (2), (8), and (37), the residual extropy of the $r$ th $m-d g o s, X_{d(r ; n, k, m)}, 1 \leq r \leq n$, is given by

$$
J\left(X_{d(r ; n, k, m)}\right)=\frac{1}{\bar{F}_{d(r ; n, k, m)}^{2}(x)} J\left(U_{d(r ; n, k, m)}\right) \mathbb{E}\left(f\left(F^{-1}\left(V_{r}\right)\right) \mid V_{r}<1-F(t)\right),
$$

where $V_{r} \sim G B 1\left(x ; m+1,\left(2 \gamma_{r}-1\right) /(m+1), 2 r-1\right)$ with PDF defined in (19) and $\bar{F}_{d(r ; n, k, m)}(x)=1-F_{d(r ; n, k, m)}(x)$ defined in (37).
Theorem 7. Let $X$ be a continuous $r . v$. that is nonnegative with CDF $F(x)$ distribution. Then, from (3), (8), and (37), the past extropy of the rth $m$-dgos, $X_{d(r ; n, k, m)}, 1 \leq r \leq n$, is given by

$$
J\left(X_{d(r ; n, k, m)}\right)=\frac{1}{F_{d(r ; n, k, m)}^{2}(x)} J\left(U_{d(r ; n, k, m)}\right) \mathbb{E}\left(f\left(F^{-1}\left(V_{r}\right)\right) \mid V_{r}>1-F(t)\right),
$$

where $V_{r} \sim G B 1\left(x ; m+1,\left(2 \gamma_{r}-1\right) /(m+1), 2 r-1\right)$ with $P D F$ defined in (19) and $F_{d(r ; n, k, m)}(x)$ defined in (37).

\section{Residual Extropy of Concomitants of m-Generalized Order Statistics}

In this section, we will discuss the residual extropy of concomitants of $m$ - gos under the FGM family. From equation (9), the conditional CDF of $Y$ given $X=x$ is given by

$$
F_{Y \mid X}(y \mid x)=F_{Y}(y)\left[1+\alpha\left(1-2 F_{X}(x)\right)\left(1-F_{Y}(y)\right)\right] .
$$

Under the FGM family with conditional CDF given by equation (42), Mohie El-Din et al. [23] presented the CDF of the concomitant of $m-\operatorname{gos} Y_{[r ; n, k, m]}, 1 \leq r \leq n$, as follows:

$$
G_{[r ; n, k, m]}(y)=F_{Y}(y)\left[1-\alpha T^{*}(r ; n, k, m)\left(1-F_{Y}(y)\right)\right],
$$

where $f_{(r ; n, k, m)}(x)$ is the PDF of $m-\operatorname{gos} X_{(r ; n, k, m)}$. Therefore,

$$
\bar{G}_{[r ; n, k, m]}(y)=1-G_{[r ; n, k, m]}(y)=1-F_{Y}(y)\left[1-\alpha T^{*}(r ; n, k, m)\left(1-F_{Y}(y)\right)\right]=\bar{F}_{Y}(y)\left[1+\alpha T^{*}(r ; n, k, m) F_{Y}(y)\right] .
$$


From (4) and (44), the residual extropy of concomitants of $m$ - gos is given by

$$
\begin{aligned}
\zeta_{[r ; n, k, m]}(Y)= & \frac{-1}{2} \int_{0}^{\infty}\left(\bar{G}_{[r ; n, k, m]}(y)\right)^{2} \mathrm{~d} y \\
= & \frac{-1}{2} \int_{0}^{\infty}\left[\bar{F}_{Y}^{2}(y)+2 \alpha T^{*}(r ; n, k, m) \bar{F}_{Y}^{2}(y) F_{Y}(y)+\left(\alpha T^{*}(r ; n, k, m)\right)^{2} \bar{F}_{Y}^{2}(y) F_{Y}^{2}(y)\right] \mathrm{d} y \\
= & \zeta(Y)+\left(\left(\alpha T^{*}(r ; n, k, m)\right)^{2}-4 \alpha T^{*}(r ; n, k, m)\right) \mathbb{E}\left(Y F_{Y}(y)\right) \\
& +3\left(\alpha T^{*}(r ; n, k, m)-\left(\alpha T^{*}(r ; n, k, m)\right)^{2}\right) \mathbb{E}\left(Y F_{Y}^{2}(y)\right) \\
& +2\left(\alpha T^{*}(r ; n, k, m)\right)^{2} \mathbb{E}\left(Y F_{Y}^{3}(y)\right)+\alpha T^{*}(r ; n, k, m) \mathbb{E}(Y) .
\end{aligned}
$$

Furthermore, we can write (45) in terms of the moments as follows:

$$
\begin{aligned}
\zeta_{[r ; n, k, m]}(Y)=\zeta(Y)+ & \frac{1}{2}\left(\left(\alpha T^{*}(r ; n, k, m)\right)^{2}-4 \alpha T^{*}(r ; n, k, m)\right) \mu_{2: 2} \\
& +\left(\alpha T^{*}(r ; n, k, m)-\left(\alpha T^{*}(r ; n, k, m)\right)^{2}\right) \mu_{3: 3} \\
& +\frac{1}{2}\left(\alpha T^{*}(r ; n, k, m)\right)^{2} \mu_{4: 4}+\alpha T^{*}(r ; n, k, m) \mu,
\end{aligned}
$$

where $\mu_{n: n}=\int_{0}^{\infty} y f_{y_{n: n}}(y) \mathrm{d} y$ and $y_{n: n}$ is the $n$th order statistic of a random sample of size $n$ of the $Y$ variate, $\mu=\mathbb{E}(Y)$.

4.1. Stop-Loss Transform and Gini Coefficient. In this section, we will present $\zeta_{[r ; n, k, m]}(Y)$ related to stop-loss transform and Gini index.

Definition 1. The stop-loss transform $Z_{F}(t)$ of the nonnegative r.v. $Y$ is defined as

$$
Z_{F}(t)=\mathbb{E}(\max \{Y-t, 0\})=\int_{t}^{\infty} \bar{F}_{Y}(y) \mathrm{d} y .
$$

Definition 2. Suppose $X$ and $Y$ are independent r.v.'s and have the same distribution as $X$. Then, the Gini index or Gini coefficient is given by

$$
G_{\text {index }}=\frac{\mathbb{E}(|X-Y|)}{\mathbb{E}(|X+Y|)}=1-\frac{\int_{0}^{\infty} \bar{F}_{X}^{2}(x) \mathrm{d} x}{\mathbb{E}(X)},
$$

(see Wang [24] for more details).
Remark 1. From Jahanshahi et al. [14], based on (4) and Definitions 1 and 2, we have

$$
\begin{aligned}
\zeta(Y) & =\frac{-1}{2}\left[\mathbb{E}(Y)-\mathbb{E}\left(Z_{F}(Y)\right)\right] \\
& =\frac{-1}{2}\left[\mathbb{E}(Y)-\mathbb{E}\left(\bar{F}_{Y}(y) m_{F}(Y)\right)\right]=\frac{\mathbb{E}(Y)}{2}\left[G_{\text {index }}-1\right],
\end{aligned}
$$

where $m_{F}(Y)=\left(Z_{F}(Y) / \bar{F}_{Y}(y)\right)$ is the mean residual life function.

Theorem 8. Let $Y$ be a continuous r.v. that is nonnegative with CREX $\zeta(X)$ defined in (4). Then, the cumulative residual extropy of concomitants of $m-g o s, Y_{(r ; n, k, m)}$, can be expressed as

$$
\begin{aligned}
\zeta_{[r ; n, k, m]}(Y) & =\frac{1}{2} \mathbb{E}\left(Z_{F}(Y)\right)+T_{[r ; n, k, m]}^{*} \\
& =\frac{1}{2} \mathbb{E}\left(\bar{F}_{Y}(y) m_{F}(Y)\right)+T_{[r ; n, k, m]}^{*} \\
& =\frac{1}{2} \mathbb{E}(Y) G_{\text {index }}+T_{[r ; n, k, m]}^{*},
\end{aligned}
$$


where

$$
\begin{array}{r}
T_{[r ; n, k, m]}^{*}=\left(\left(\alpha T^{*}(r ; n, k, m)\right)^{2}-4 \alpha T^{*}(r ; n, k, m)\right) \mathbb{E}\left(Y F_{Y}(y)\right)+3\left(\alpha T^{*}(r ; n, k, m)-\left(\alpha T^{*}(r ; n, k, m)\right)^{2}\right) \mathbb{E}\left(Y F_{Y}^{2}(y)\right) \\
+2\left(\alpha T^{*}(r ; n, k, m)\right)^{2} \mathbb{E}\left(Y F_{Y}^{3}(y)\right)+\alpha T^{*}(r ; n, k, m) \mathbb{E}(Y) .
\end{array}
$$

Proof. The proof directly follows from (45) and Remark 1.

4.2. Upper Bound of $\zeta_{[r ; n, k, m]}(Y)$. In another view of the cumulative residual extropy of concomitants of $m$-gos, $\zeta_{[r ; n, k, m]}(Y)$, we present it depending on its upper bound.

Theorem 9. Let $Y$ be a continuous r.v. that is nonnegative with survival function $\bar{F}_{Y}(y)$. Based on FGM family with
CDF given by (9), utilizing (4), the residual extropy of the rth concomitants $m$ - gos, $Y_{[r ; n, k, m]}, 1 \leq r \leq n$, can be expressed by

$$
\begin{aligned}
\zeta_{[r ; n, k, m]}(Y) & \leq \zeta(y)+\alpha T^{*}(r ; n, k, m)\left[3 \mathbb{E}\left(Y F_{Y}^{2}(y)\right)\right. \\
& \left.+\mathbb{E}(Y)-4 \mathbb{E}\left(Y F_{Y}(y)\right)\right] .
\end{aligned}
$$

Proof. Using Bernoulli's inequality, from (44), we have

$$
\begin{aligned}
\zeta_{[r ; n, k, m]}(Y)= & \frac{-1}{2} \int_{0}^{\infty}\left(\bar{G}_{[r ; n, k, m]}(y)\right)^{2} \mathrm{~d} y \\
& =\frac{-1}{2} \int_{0}^{\infty} \bar{F}_{Y}^{2}(y)\left[1+\alpha T^{*}(r ; n, k, m) F_{Y}(y)\right]^{2} \mathrm{~d} y \\
& \leq \frac{-1}{2} \int_{0}^{\infty} \bar{F}_{Y}^{2}(y)\left[1+2 \alpha T^{*}(r ; n, k, m) F_{Y}(y)\right] \mathrm{d} y \\
= & \zeta(y)+\alpha T^{*}(r ; n, k, m)\left[3 \mathbb{E}\left(Y F_{Y}^{2}(y)\right)+\mathbb{E}(Y)-4 \mathbb{E}\left(Y F_{Y}(y)\right)\right],
\end{aligned}
$$

where $T^{*}(r ; n, k, m)$ is defined in (12).

From the previous theorem, we can conclude the following remark.

Remark 2. Since $-1<T^{*}(r ; n, k, m)<1$, then the residual extropy of the concomitant of $r$ th $m$ - gos given in (53) can be considered in following cases:

(1) If $T^{*}(r ; n, k, m)=0$, then $\zeta_{[r ; n, k, m]}(Y)=\zeta(y)$.

(2) If $0<\alpha<1$ and $0<T^{*}(r ; n, k, m)<1$ or $-1<\alpha<0$ and $-1<T^{*}(r ; n, k, m)<0$, then

$$
\zeta_{[r ; n, k, m]}(Y)<\zeta(y)+\alpha T^{*}(r ; n, k, m)\left[3 \mathbb{E}\left(Y F_{Y}^{2}(y)\right)+\mathbb{E}(Y)\right] \text {. }
$$

(3) If $0<\alpha<1$ and $-1<T^{*}(r ; n, k, m)<0$ or $-1<\alpha<0$ and $0<T^{*}(r ; n, k, m)<1$, then

$$
\zeta_{[r ; n, k, m]}(Y)<\zeta(y)-4 \alpha T^{*}(r ; n, k, m) \mathbb{E}\left(Y F_{Y}(y)\right)
$$

Theorem 10. Let $Y$ be a continuous r.v. that is nonnegative with survival function $\bar{F}_{Y}(y)$. Then, we have the following:

(1) From Remark 2, under the conditions on the parameters in (54), we get

$$
\zeta_{[r ; n, k, m]}(Y)<\frac{1}{2} \xi(Y)+F_{[r ; n, k, m]}^{*} \leq \frac{\mathbb{E}\left(Y^{2}\right)}{4 \mathbb{E}(Y)}+F_{[r ; n, k, m]}^{*} .
$$

(2) From Remark 2, under the conditions on the parameters in (55), we get

$$
\zeta_{[r ; n, k, m]}(Y)<\frac{1}{2} \xi(Y)+R_{[r ; n, k, m]}^{*} \leq \frac{\mathbb{E}\left(Y^{2}\right)}{4 \mathbb{E}(Y)}+R_{[r ; n, k, m]}^{*},
$$

where $\xi(Y)=-\int_{0}^{\infty} \bar{F}_{Y}(y) \log \bar{F}_{Y}(y) d y$ is the cumulative residual entropy presented by Rao et al. [25]:

$$
\begin{aligned}
F_{[r ; n, k, m]}^{*}= & 3 \alpha T^{*}(r ; n, k, m) \mathbb{E}\left(\mathrm{YF}_{Y}^{2}(y)\right) \\
& +\mathbb{E}(Y)\left(\alpha T^{*}(r ; n, k, m)-\frac{1}{2}\right), \\
R_{[r ; n, k, m]}^{*}= & -4 \alpha T^{*}(r ; n, k, m) \mathbb{E}\left(\mathrm{YF}_{Y}(y)\right)-\frac{1}{2} \mathbb{E}(Y) .
\end{aligned}
$$

Proof. The proof directly follows from inequality $-\log x \geq$ $(1-x), x>0$ and $\xi(Y) \leq\left(\mathbb{E}\left(Y^{2}\right) / 2 \mathbb{E}(Y)\right)$; see Rao et al. [25]. 
In the next remark, we will give an application of the previous results, taking the order statistics (with $k=1$ and $m=0)$ as a special case under the FGM family.

Remark 3. For order statistic ( $m=0$ and $k=1)$, we have $T^{*}(r ; n, 1,0)=-(n-2 r+1) /(n+1)$. Moreover, by controlling the values of $n$ and $r$, we can observe the following:

(1) $T^{*}(r ; n, 1,0)=0$, if $n$ is odd and $r=(n+1) / 2$

(2) $T^{*}(r ; n+1,1,0)=0$, if $n$ is even, $r=(n / 2)+1$ and $n$ is replaced with $n+1$

(3) $T^{*}(r ; n, 1,0)<(>) 0$, if $n$ is even and $r<(>)(n / 2)$

(4) $T^{*}(r ; n, 1,0)<(>) 0$, if $n$ is odd and $r<(>)(n+1) / 2$

Example 3. Let $Y$ be a continuous r.v. that is nonnegative arising from $\operatorname{EXP}(\lambda)$ with $\operatorname{CDF}$ defined in (22); then, utilizing (58) and (59), we have

$$
\begin{aligned}
\zeta_{[r ; n, k, m]}(Y) & =-\frac{6+\alpha T^{*}(r ; n, k, m)\left(4+\alpha T^{*}(r ; n, k, m)\right)}{24 \lambda} \\
\frac{1}{2} \xi(Y)+F_{[r ; n, k, m]}^{*} & =\frac{17 \alpha T^{*}(r ; n, k, m)}{6 \lambda}=\frac{\mathbb{E}\left(Y^{2}\right)}{4 \mathbb{E}(Y)}+F_{[r ; n, k, m]}^{*} \\
\frac{1}{2} \xi(Y)+R_{[r ; n, k, m]}^{*} & =\frac{-3 \alpha T^{*}(r ; n, k, m)}{\lambda}=\frac{\mathbb{E}\left(Y^{2}\right)}{4 \mathbb{E}(Y)}+R_{[r ; n, k, m]}^{*}
\end{aligned}
$$

For order statistics (with $m=0$ and $k=1$ ), we can apply some numerical values as follows:

(1) For $n=20, r=13, \alpha=0.4$, and $\lambda=1$, we have $T^{*}(r ; n, 1,0)=(5 / 21), \zeta_{[r ; n, 1,0]}(Y)=-0.266251$, and $(1 / 2) \xi(Y)+F_{[r ; n, 1,0]}^{*}=0.26984=\left(\mathbb{E}\left(Y^{2}\right) / 4 \mathbb{E}(Y)\right)+$ $F_{[r ; n, 1,0]}^{*}$

(2) For $n=27, r=13, \alpha=0.3$, and $\lambda=1$, we have $T^{*}(r ; n, 1,0)=(-1 / 14), \quad \zeta_{[r ; n, 1,0]}(Y)=-0.246448$, and $(1 / 2) \xi(Y)$

$$
+R_{[r ; n, 1,0]}^{*}=0.06428=\left(\mathbb{E}\left(Y^{2}\right) / 4 \mathbb{E}(Y)\right)+R_{[r ; n, 1,0]}^{*}
$$

which assure Theorem 10.

Example 4. Let $Y$ be a continuous r.v. that is nonnegative arising from uniform distribution $U(0, b), b>0$; then, utilizing (58) and (59), we have

$$
\begin{aligned}
\zeta_{[r ; n, k, m]}(Y) & =\frac{-b}{60}\left(10+5 \alpha T^{*}(r ; n, k, m)+\left(\alpha T^{*}(r ; n, k, m)\right)^{2}\right) \\
\frac{1}{2} \xi(Y)+F_{[r ; n, k, m]}^{*} & =\frac{b}{8}\left(-1+10 \alpha T^{*}(r ; n, k, m)\right) \\
\frac{\mathbb{E}\left(Y^{2}\right)}{4 \mathbb{E}(Y)}+F_{[r ; n, k, m]}^{*} & =\frac{b}{12}\left(-1+15 \alpha T^{*}(r ; n, k, m)\right) \\
\frac{1}{2} \xi(Y)+R_{[r ; n, k, m]}^{*} & =\frac{-b}{24}\left(3+32 \alpha T^{*}(r ; n, k, m)\right) \\
\frac{\mathbb{E}\left(Y^{2}\right)}{4 \mathbb{E}(Y)}+R_{[r ; n, k, m]}^{*} & =\frac{-b}{12}\left(1+16 \alpha T^{*}(r ; n, k, m)\right)
\end{aligned}
$$

For order statistics (with $m=0$ and $k=1$ ), we can apply some numerical values as follows:

(1) For $n=20, r=13, \alpha=0.4$, and $b=1$, we have $T^{*}(r ; n, 1,0)=(5 / 21), \quad \zeta_{[r ; n, 1,0]}(Y)=-0.174754$, $(1 / 2) \xi(Y)+F_{[r ; n, 1,0]}^{*}=-0.005952$, and $\left(\mathbb{E}\left(Y^{2}\right) / 4 \mathbb{E}\right.$ $(Y))+F_{[r ; n, 1,0]}^{*}=0.0357$

(2) For $n=27, r=13, \alpha=0.3$, and $b=1$, we have $T^{*}(r ; n, 1,0)=(-1 / 14), \quad \zeta_{[r ; n, 1,0]}(Y)=-0.164889$, and $(1 / 2) \xi(Y)+R_{[r ; n, 1,0]}$

$$
\begin{aligned}
& *=-0.0964286,\left(\mathbb{E}\left(Y^{2}\right) / 4 \mathbb{E}(Y)\right)+R_{[r ; n, 1,0]}^{*}= \\
& -0.0547619
\end{aligned}
$$

Therefore, (1.) and (2.) assure Theorem 10.

Theorem 11. Let $Y$ be a continuous r.v. that is nonnegative with survival function $\bar{F}_{Y}(y)$. From Remark 2, under the conditions on the parameters in (54), for order statistics (with $m=0$ and $k=1$ ) as a special case of $m-g o s$, we denote 


$$
\zeta_{[r ; n, 1,0]}(Y)<\zeta(Y)+\alpha T^{*}(r ; n, 1,0)\left[s_{3}+\mu\right]=Z_{(r ; n, 1,0)}^{*}(y),
$$

where $s_{i}=m_{i}+i \int_{m_{i}}^{\infty} \bar{F}_{Y}(y) d y, m_{i}=\inf \left\{y: \bar{F}_{Y}(y) \leq(1 / i)\right\}$, $i=1,2, \ldots, n$.

Proof. A method of obtaining an upper bound for the mean of the maximum of $n$ identically distributed nonnegative r.v.'s is given by Lai and Robbins [26] and Gravey [27]. If $\mathbb{E}\left(Y_{i}^{+}\right)<\infty, i=1,2, \ldots, n$, then

$$
\mu_{n: n}=\mathbb{E}\left(Y_{n: n}\right) \leq m_{n}+n \int_{m_{n}}^{\infty} \bar{F}_{X}(x) \mathrm{d} x=s_{n} .
$$

Thus, from (54),

$$
\begin{array}{r}
\zeta_{[r ; n, 1,0]}(Y)<\zeta(y)+\alpha T^{*}(r ; n, 1,0)\left[3 \mathbb{E}\left(Y F_{Y}^{2}(y)\right)+\mathbb{E}(Y)\right] \\
=\zeta(y)+\alpha T^{*}(r ; n, 1,0)\left[\mu_{3: 3}+\mu\right] \\
<\zeta(Y)+\alpha T^{*}(r ; n, 1,0)\left[s_{3}+\mu\right]=Z_{(r ; n, 1,0)}^{*}(y) .
\end{array}
$$

Example 5. Suppose $Y$ follows $\operatorname{EXP}(1)$ with survival function $\bar{F}_{Y}(y)=e^{-y}, 0<y$. From (63), we have $\mu_{n: n} \leq 1+\log n$. Therefore, using (62) and from Remark 2, under the conditions on the parameters in (54), with $n=50, r=28, \alpha=0.3$, and $T^{*}(r ; n, 1,0)=(5 / 51)$, we have $\zeta_{[r ; n, 1,0]}(Y)=-0.25493$ and $Z_{(r ; n, 1,0)}^{*}(y)=-0.15886$, which assure the previous results.

\subsection{Stochastic Orders}

Definition 3. $Y_{2}$ is known to be smaller than $Y_{1}$ in the usual stochastic order, denoted by $Y_{2} \leq_{\mathrm{st}} Y_{1}$ if and only if $\bar{F}_{Y_{2}}(t) \leq \bar{F}_{Y_{1}}(t)$, for all $t \in \mathbb{R}$. For more details, see Shaked and Shanthikumar [28].

Theorem 12. Let $Y_{1}$ and $Y_{2}$ be two nonnegative continuous r.v.'s with CDF's $F_{Y_{1}}(\cdot)$ and $F_{Y_{2}}(\cdot)$ and finite mean $\mathbb{E}\left(Y_{1}\right)$ and $\mathbb{E}\left(Y_{2}\right)$, respectively. If $Y_{2} \leq_{s t} Y_{1}$, then we have the following:

(1) From Remark 2, under the conditions on the parameters in (54), we get

$$
\begin{aligned}
\zeta_{[r ; n, k, m]}\left(Y_{1}\right) & -\zeta_{[r ; n, k, m]}\left(Y_{2}\right) \\
& \leq \alpha T^{*}(r ; n, k, m)\left[\mathbb{E}\left(Y_{1}\right)-\mathbb{E}\left(Y_{2}\right)\right] .
\end{aligned}
$$

(2) From Remark 2, under the conditions on the parameters in (55), we get

$$
\zeta_{[r ; n, k, m]}\left(Y_{1}\right)-\zeta_{[r ; n, k, m]}\left(Y_{2}\right) \leq \zeta\left(Y_{1}\right)-\zeta\left(Y_{2}\right)
$$

Proof. First, from Theorem 7 of Jahanshahi et al. [14], if $Y_{2} \leq_{\text {st }} Y_{1}$, then $\zeta\left(Y_{2}\right) \geq \zeta\left(Y_{1}\right)$. Therefore, the proof of (1) is

$$
\begin{aligned}
& \zeta_{[r ; n, k, m]}\left(Y_{1}\right)-\zeta_{[r ; n, k, m]}\left(Y_{2}\right) \leq \zeta\left(Y_{1}\right)-\zeta\left(Y_{2}\right) \\
& +3 \alpha T^{*}(r ; n, k, m)\left[\int_{0}^{\infty} t\left[F_{Y_{1}}^{2}(t) f_{Y_{1}}(t)-F_{Y_{2}}^{2}(t) f_{Y_{2}}(t)\right] \mathrm{d} t\right] \\
& +\alpha T^{*}(r ; n, k, m)\left[\mathbb{E}\left(Y_{1}\right)-\mathbb{E}\left(Y_{2}\right)\right] \\
& \leq \alpha T^{*}(r ; n, k, m)\left[\mathbb{E}\left(Y_{1}\right)-\mathbb{E}\left(Y_{2}\right)\right] .
\end{aligned}
$$

The proof of (2) is

$$
\begin{aligned}
& \zeta_{[r ; n, k, m]}\left(Y_{1}\right)-\zeta_{[r ; n, k, m]}\left(Y_{2}\right) \leq \zeta\left(Y_{1}\right)-\zeta\left(Y_{2}\right) \\
& -4 \alpha T^{*}(r ; n, k, m)\left[\int_{0}^{\infty} t\left[F_{Y_{1}}(t) f_{Y_{1}}(t)-F_{Y_{2}}(t) f_{Y_{2}}(t)\right] \mathrm{d} t\right] \\
& \leq \zeta\left(Y_{1}\right)-\zeta\left(Y_{2}\right) .
\end{aligned}
$$

Now, we will give an application of the last theorem as follows.

Example 6. Let $Y_{1}$ and $Y_{2}$ be two r.v.'s of power function distribution with CDF's $F_{Y_{1}}(y)=y^{2}, 0<y<1$ and $F_{Y_{2}}(y)=y, 0<y<1$, respectively. For order statistics (with $m=0$ and $k=1$ ). From Theorem 12, we have the following:

(1) For $n=20, \quad r=12$, and $\alpha=0.6$, we have $T^{*}(r ; n, 1,0)=(1 / 7), \zeta_{[r ; n, 1,0]}\left(Y_{1}\right)-\zeta_{[r ; n, 1,0]}\left(Y_{2}\right)=-$ 0.09935 , and $\alpha T^{*}(r ; n, k, m)\left[\mathbb{E}\left(Y_{1}\right)-\mathbb{E}\left(Y_{2}\right)\right]=$ 0.01428

(2) For $n=20, \quad r=8$, and $\alpha=0.8$, we have $T^{*}(r ; n, 1,0)=(-5 / 21)$, $\zeta_{[r ; n, 1,0]}\left(Y_{1}\right)-\zeta_{[r ; n, 1,0]}\left(Y_{2}\right)=-0.10121$, and $\zeta\left(Y_{1}\right)-$ $\zeta\left(Y_{2}\right)=-0.1$

which assure the previous results.

\section{Nonparametric Estimation}

In this section, we obtain a nonparametric estimation of the residual extropy of concomitants of $m$ - gos under the FGM family by the empirical data. Let $Y_{1}, \ldots, Y_{n}$ be a random sample from a population with CDF $F$ and its empirical estimator $F_{n}$. From (45), the empirical residual extropy of concomitants of $m-$ gos is given by

$$
\begin{aligned}
\zeta_{[r ; n, k, m]}\left(F_{n}\right) & =\frac{-1}{2} \int_{0}^{\infty} \bar{F}_{n}^{2}(y)\left(1+\alpha T^{*}(r ; n, k, m) F_{n}(y)\right)^{2} \mathrm{~d} y \\
& =\frac{-1}{2} \sum_{j=1}^{n-1} \int_{Y_{(j)}}^{Y_{(j+1)}} \bar{F}_{n}^{2}(y)\left(1+\alpha T^{*}(r ; n, k, m) F_{n}(y)\right)^{2} \mathrm{~d} y,
\end{aligned}
$$

where $\bar{F}_{n}(y)=1-F_{n}(y), F_{n}(y)$ is the empirical CDF, and $Y_{(1)} \leq Y_{(2)} \leq \cdots \leq Y_{(n)}$ are the associated order statistics of the random sample.

To estimate $\zeta_{[r ; n, k, m]}$, we consider the first empirical estimator $\zeta_{1[r ; n, k, m]}\left(F_{n}\right)$ as follows: 


$$
\begin{aligned}
& \zeta_{1[r ; n, k, m]}\left(F_{n}\right) \\
& =\frac{-1}{2} \sum_{j=1}^{n-1} W_{j+1}\left(1-\frac{j}{n}\right)^{2}\left(1+\alpha T^{*}(r ; n, k, m) \frac{j}{n}\right)^{2},
\end{aligned}
$$

where $\quad W_{j+1}=Y_{(j+1)}-Y_{(j)}, \quad F_{n}(y)=(j / n)$, $j=1,2, \ldots, n-1$, and $W_{1}=Y_{(1)}$. Moreover, the second empirical estimator (kernel-smoothed estimator) $\zeta_{2[r ; n, k, m]}\left(F_{n}\right)$ is given by

$$
\begin{aligned}
\zeta_{2[r ; n, k, m]}\left(F_{n}\right)= & \frac{-1}{2} \sum_{j=1}^{n-1} W_{j+1}\left(1-F_{n}\left(y_{j}\right)\right)^{2} \\
& \cdot\left(1+\alpha T^{*}(r ; n, k, m) F_{n}\left(y_{j}\right)\right)^{2},
\end{aligned}
$$

where

$$
F_{n}\left(y_{j}\right)=\frac{1}{n} \sum_{i=1}^{n} h\left(\frac{y-Y_{i}}{l}\right),
$$

$h(y)=\int_{-\infty}^{y} K(t) \mathrm{d} t$ and $l$ is a bandwidth parameter; see Nadaraya [29].

In the following examples, we apply the proposed methods to explain the performance of the empirical and kernel estimators.

Example 7. Let $X_{1}, \ldots, X_{n}$ be a random sample of uniform distribution $U(0,1)$. According to Pyke [30], the sample spacing $W_{j+1}$ follows the beta distribution $\operatorname{Beta}(1, n)$. Hence, from (70) and (71), we have

$$
\begin{aligned}
\mathbb{E}\left(\zeta_{1[r ; n, k, m]}\left(F_{n}\right)\right) & =\frac{-1}{2(n+1)} \sum_{j=1}^{n-1}\left(1-\frac{j}{n}\right)^{2}\left(1+\alpha T^{*}(r ; n, k, m) \frac{j}{n}\right)^{2} \\
\operatorname{Var}\left(\zeta_{1[r ; n, k, m]}\left(F_{n}\right)\right) & =\frac{n}{4(n+1)^{2}(n+2)} \sum_{j=1}^{n-1}\left(1-\frac{j}{n}\right)^{4}\left(1+\alpha T^{*}(r ; n, k, m) \frac{j}{n}\right)^{4}, \\
\mathbb{E}\left(\zeta_{2[r ; n, k, m]}\left(F_{n}\right)\right) & =\frac{-1}{2(n+1)} \sum_{j=1}^{n-1}\left(1-F_{n}\left(y_{j}\right)\right)^{2}\left(1+\alpha T^{*}(r ; n, k, m) F_{n}\left(y_{j}\right)\right)^{2}, \\
\operatorname{Var}\left(\zeta_{2[r ; n, k, m]}\left(F_{n}\right)\right) & =\frac{n}{4(n+1)^{2}(n+2)} \sum_{j=1}^{n-1}\left(1-F_{n}\left(y_{j}\right)\right)^{4}\left(1+\alpha T^{*}(r ; n, k, m) F_{n}\left(y_{j}\right)\right)^{4} .
\end{aligned}
$$

Example 8. Let $X_{1}, \ldots, X_{n}$ be a random sample of $\operatorname{Exp}(\lambda)$. According to Pyke [30], the sample spacing
$W_{j+1}$ follows $\operatorname{Exp}(\lambda(n-j))$. Hence, from (70) and (71), we have

$$
\begin{aligned}
\mathbb{E}\left(\zeta_{1[r ; n, k, m]}\left(F_{n}\right)\right) & =\frac{-1}{2 n \lambda} \sum_{j=1}^{n-1}\left(1-\frac{j}{n}\right)\left(1+\alpha T^{*}(r ; n, k, m) \frac{j}{n}\right)^{2}, \\
\operatorname{Var}\left(\zeta_{1[r ; n, k, m]}\left(F_{n}\right)\right) & =\frac{1}{4 n^{2} \lambda^{2}} \sum_{j=1}^{n-1}\left(1-\frac{j}{n}\right)^{2}\left(1+\alpha T^{*}(r ; n, k, m) \frac{j}{n}\right)^{4}, \\
\mathbb{E}\left(\zeta_{2[r ; n, k, m]}\left(F_{n}\right)\right) & =\frac{-1}{2 \lambda} \sum_{j=1}^{n-1} \frac{\left(1-F_{n}\left(y_{j}\right)\right)^{2}}{n-j}\left(1+\alpha T^{*}(r ; n, k, m) F_{n}\left(y_{j}\right)\right)^{2}, \\
\operatorname{Var}\left(\zeta_{2[r ; n, k, m]}\left(F_{n}\right)\right) & =\frac{1}{4 \lambda^{2}} \sum_{j=1}^{n-1} \frac{\left(1-F_{n}\left(y_{j}\right)\right)^{4}}{(n-j)^{2}}\left(1+\alpha T^{*}(r ; n, k, m) F_{n}\left(y_{j}\right)\right)^{4} .
\end{aligned}
$$

Based on order statistics $(k=1, m=0)$, Table 1 presents the mean and variance of $\zeta_{1[r ; n, 1,0]}$ and $\zeta_{2[r ; n, 1,0]}$ from $U(0,1)$ and $\operatorname{EXP}(1)$, respectively, by using different values of sample size $(n=10,30,50,70,100)$. In Table 1 , for fixed $n$ and $r$ increases, we conclude that the mean decreases and the variance increases. 
TABLE 1: Mean and variance of the empirical residual extropy of concomitants of order statistics, $\alpha=0.8$.

\begin{tabular}{|c|c|c|c|c|c|c|c|c|c|}
\hline \multirow{3}{*}{$n$} & \multirow{3}{*}{$r$} & \multicolumn{4}{|c|}{$U(0,1)$ distribution } & \multicolumn{4}{|c|}{ EXP(1) distribution } \\
\hline & & \multicolumn{2}{|c|}{ The first estimator } & \multicolumn{2}{|c|}{ The second estimator } & \multicolumn{2}{|c|}{ The first estimator } & \multicolumn{2}{|c|}{ The second estimator } \\
\hline & & $\mathbb{E}\left(\zeta_{1[r ; n, 1,0]}\right)$ & $\operatorname{Var}\left(\zeta_{2[r ; n, 1,0]}\right)$ & $\mathbb{E}\left(\zeta_{2[r ; n, 1,0]}\right)$ & $\operatorname{Var}\left(\zeta_{2[r ; n, 1,0]}\right)$ & $\mathbb{E}\left(\zeta_{1[r ; n, 1,0]}\right)$ & $\operatorname{Var}\left(\zeta_{2[r ; n, 1,0]}\right)$ & $\mathbb{E}\left(\zeta_{2[r ; n, 1,0]}\right)$ & $\operatorname{Var}\left(\zeta_{2[r ; n, 1,0]}\right)$ \\
\hline \multirow{3}{*}{10} & 3 & -0.1042 & 0.0019 & -0.1043 & 0.0013 & -0.1704 & 0.0047 & -0.2009 & 0.0057 \\
\hline & 5 & -0.1241 & 0.0024 & -0.1303 & 0.0019 & -0.2132 & 0.0065 & -0.2528 & 0.0084 \\
\hline & 8 & -0.1588 & 0.0035 & -0.17507 & 0.0035 & -0.2904 & 0.0108 & -0.3463 & 0.0145 \\
\hline \multirow{3}{*}{30} & 10 & -0.1317 & 0.00109 & -0.13301 & 0.000998 & -0.1977 & 0.0019 & -0.2089 & 0.0019 \\
\hline & 15 & -0.1512 & 0.001319 & -0.1544 & 0.00125 & -0.2373 & 0.0025 & -0.2552 & 0.0027 \\
\hline & 25 & -0.1966 & 0.00193 & -0.2039 & 0.00197 & -0.3333 & 0.00447 & -0.3663 & 0.0051 \\
\hline \multirow{3}{*}{50} & 15 & -0.1334 & 0.000702 & -0.13406 & 0.000667 & -0.1946 & 0.00116 & -0.20008 & 0.001106 \\
\hline & 25 & -0.1572 & 0.00086 & -0.15906 & 0.00085 & -0.2423 & 0.0015 & -0.2539 & 0.0016 \\
\hline & 40 & -0.19906 & 0.001219 & -0.2026 & 0.00123 & -0.3294 & 0.0026 & -0.3514 & 0.0028 \\
\hline \multirow{3}{*}{70} & 20 & -0.1341 & 0.000515 & -0.1343 & 0.00049 & -0.1933 & 0.00082 & -0.1964 & 0.00078 \\
\hline & 35 & -0.1598 & 0.00064 & -0.1609 & 0.00063 & -0.2445 & 0.00115 & -0.2526 & 0.00116 \\
\hline & 60 & -0.2111 & 0.00096 & -0.2137 & 0.00097 & -0.3511 & 0.00208 & -0.3685 & 0.0022 \\
\hline \multirow{3}{*}{100} & 20 & -0.1265 & 0.00034 & -0.1259 & 0.00032 & -0.1767 & 0.00051 & -0.1776 & 0.00048 \\
\hline & 50 & -0.1618 & 0.00046 & -0.1623 & 0.00045 & -0.2461 & 0.000814 & -0.2516 & 0.000819 \\
\hline & 90 & -0.2206 & 0.00073 & -0.2226 & 0.00073 & -0.36809 & 0.00158 & -0.3807 & 0.0016 \\
\hline
\end{tabular}

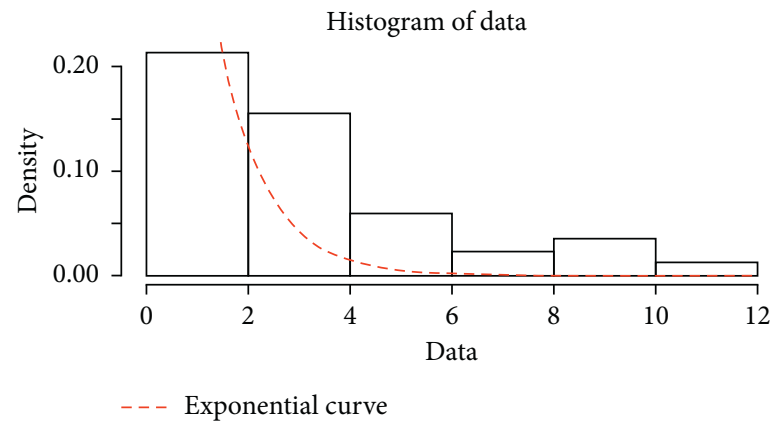

Figure 1: Histogram of serious cases of infected COVID-19 virus data.

5.1. Data Application. In the following, we illustrate our empirical estimators in real and simulated data for $\operatorname{EXP}(1)$.

Example 9. We refer to Kasilingam et al.'s [31] research to understand the spreading patterns of the COVID-19 virus; they used exponential growth modelling and identifies countries that have shown early signs of containment until 26th of March 2020. The data represent the percentage of serious cases of infections in 42 countries listed as follows: 1.56, 8.51, 2.17, 0.37, 1.09, $9.84,4.95,3.18,11.37,2.81,6.22,1.87,0.00,0.00,9.05$,
$2.44,1.38,4.17,3.74,1.37,2.33,7.80,2.10,0.47,2.54,0.92$, $0.09,0.18,1.72,1.02,0.62,2.34,0.50,2.37,3.65,0.59$, $5.76,2.14,0.88,0.95,4.17$, and 2.25 .

We use Kolmogorov-Smirnov $(K-S)$ test to check the fitting of the data for $\operatorname{EXP}(1)$, which implies that the $K-S$ statistic is 0.076282 with $p$ value 0.9674 . Thus, it is admitted to fit the data by $\operatorname{EXP}(1)$; furthermore, see Figure 1. Based on $\operatorname{EXP}(1)$, Figures 2 and 3 present the real-life and simulated data, respectively. Therefore, we can conclude that by decreasing $\alpha$ and increasing $r$, the empirical estimators approach the theoretical value and vice versa. 


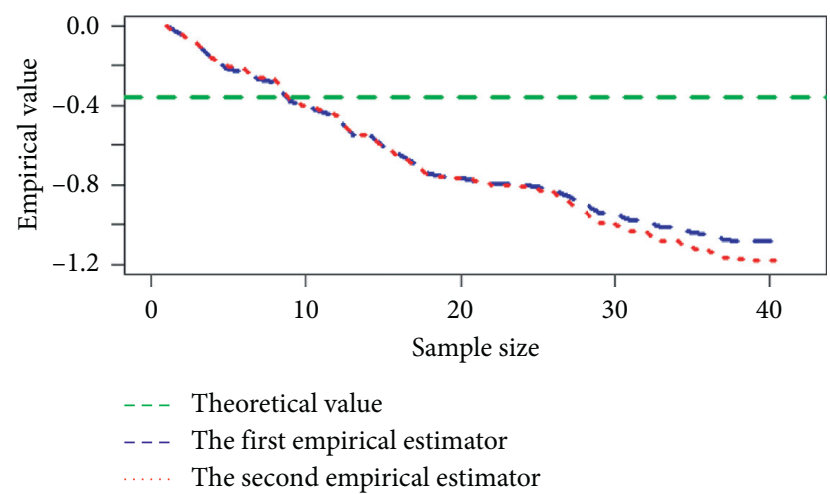

(a)

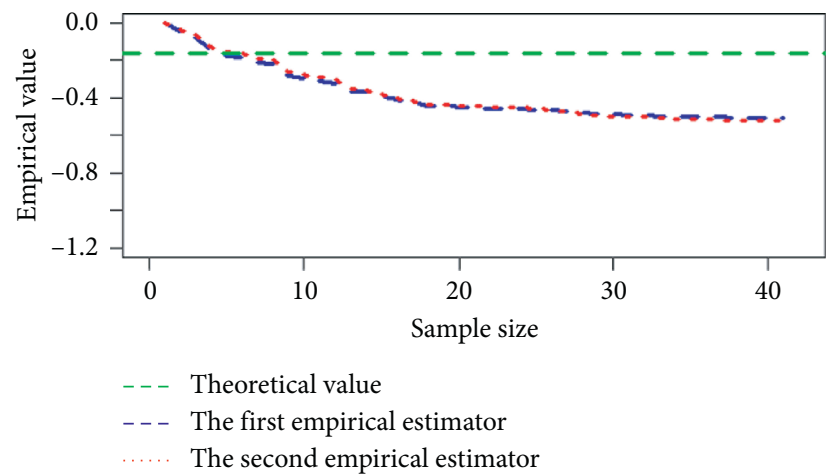

(c)

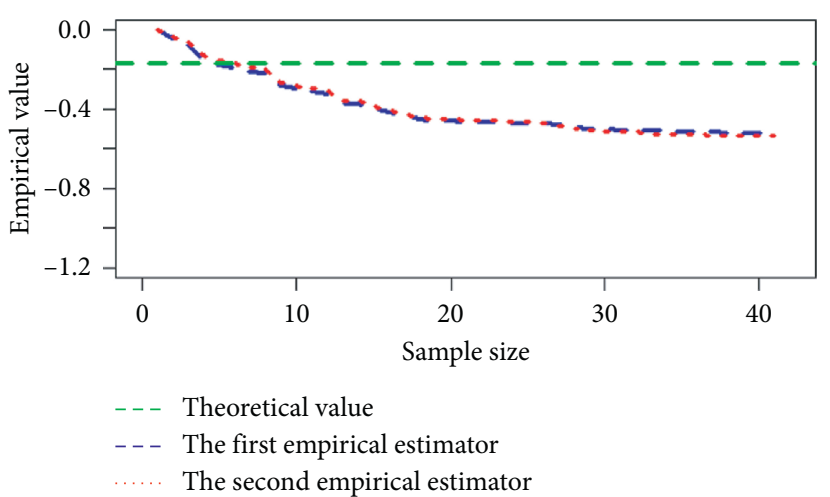

(b)

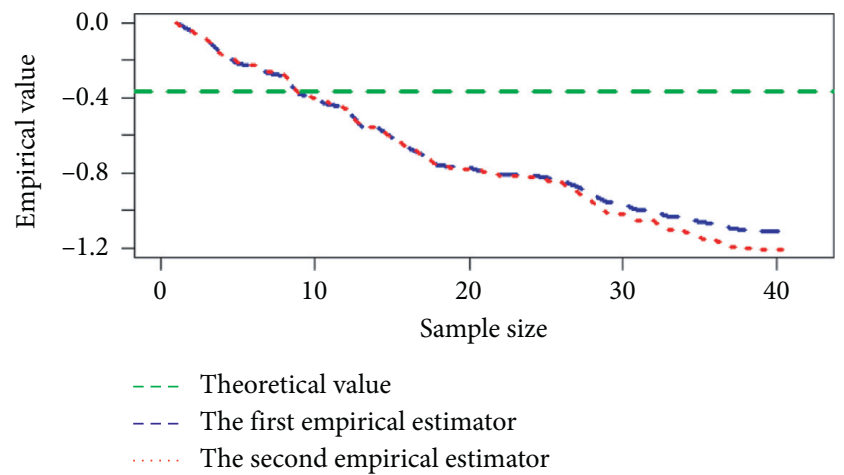

(d)

FiguRE 2: Empirical estimators of serious cases of infected COVID-19 virus data. (a) $\alpha=0.8, n=40, r=35$. (b) $\alpha=-0.8, n=40, r=35$. (c) $\alpha=0.8, n=40, r=10$. (d) $\alpha=-0.8, n=40, r=10$.

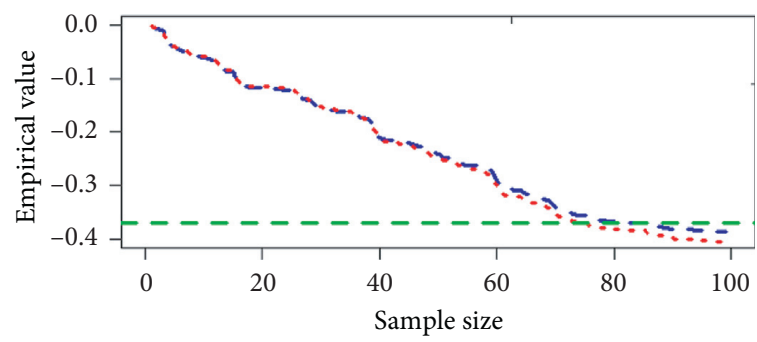

_._. Theoretical value

- - - The first empirical estimator

The second empirical estimator

(a)

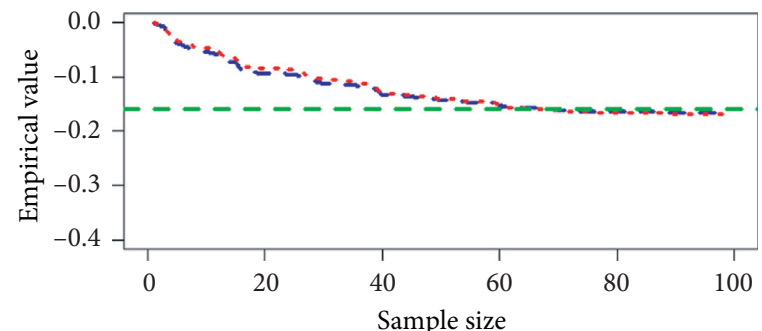

-_- Theoretical value

- - - The first empirical estimator

The second empirical estimator

(c)

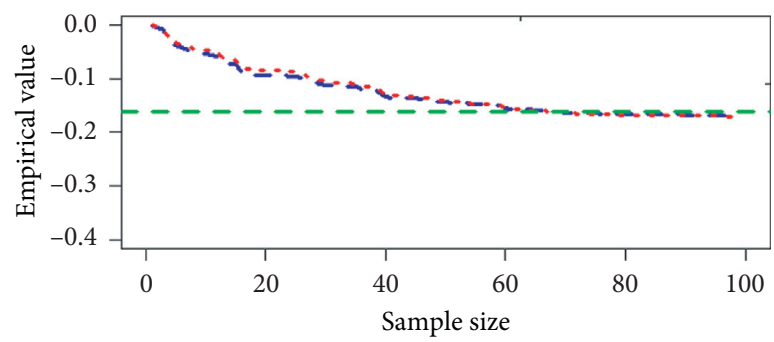

- _ Theoretical value

- - - The first empirical estimator

The second empirical estimator

(b)

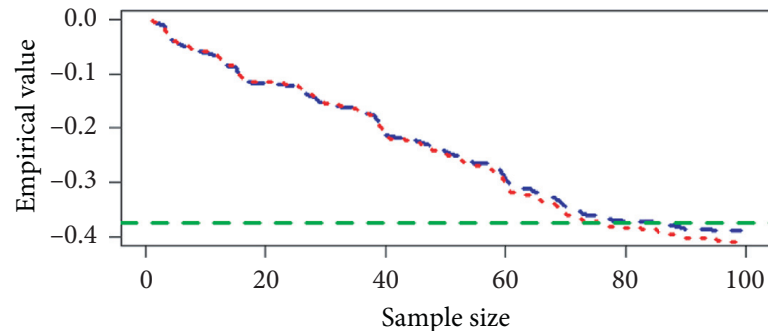

-_. Theoretical value

- - - The first empirical estimator

The second empirical estimator

(d)

FiguRE 3: Empirical estimators of simulated data. (a) $\alpha=0.8, n=100, r=90$. (b) $\alpha=-0.8, n=100, r=90$. (c) $\alpha=0.8, n=100, r=10$. (d) $\alpha=-0.8, n=100, r=10$. 


\section{Conclusion}

In this communication, we introduced the extropy of $m$ - gos and $m$ - dgos arising from any distribution and wrote them in terms of the extropy of $m-$ gos and $m-\operatorname{dgos}$ of $U(0,1)$ distribution, respectively. Furthermore, examples of the obtained models for exponential and Pareto distributions are provided. Also, we produced the lower bound of the extropy of $m$ - gos emerging from any continuous distribution in terms of the extropy of $m$ - gos of $U(0,1)$ distribution and the mode of the parent PDF. Moreover, residual and past extropy of $m-$ gos and $m-$ dgos are derived. Meanwhile, the residual extropy of concomitants of $m-$ gos, $\zeta_{[r ; n, k, m]}(Y)$, of FGM distribution is presented. The measure $\zeta_{[r ; n, k, m]}(Y)$ is discussed in terms of stop-loss transform and Gini coefficient. An alternative view of $\zeta_{[r ; n, k, m]}(Y)$ depending on its upper bound is considered. Besides, some examples and numerical results and stochastic orders of $\zeta_{[r ; n, k, m]}(Y)$ are obtained. Finally, we considered the problem of estimating $\zeta_{[r ; n, k, m]}(Y)$ by proposing two different empirical estimators of CDF. We concluded that the proposed estimators are affected by sample size $n, r$, and $\alpha$ and generally the first empirical estimator is more accurate than the second estimator.

\section{Data Availability}

All the data sets are provided within the main body of the paper.

\section{Conflicts of Interest}

The authors declare that they have no conflicts of interest.

\section{References}

[1] C. E. Shannon, "A mathematical theory of communication," Bell System Technical Journal, vol. 27, no. 3, pp. 379-423, 1948.

[2] F. Lad, G. Sanfilippo, and G. Agro, "Extropy: complementary dual of entropy," Statistical Science, vol. 30, no. 1, pp. 40-58, 2015.

[3] G. Qiu, "The extropy of order statistics and record values," Statistics and Probability Letters, vol. 120, pp. 52-60, 2017.

[4] G. Qiu and K. Jia, "The residual extropy of order statistics," Statistics and Probability Letters, vol. 133, pp. 15-22, 2018a.

[5] G. Qiu, L. Wang, and X. Wang, "On extropy properties of mixed systems," Probability in the Engineering and Informational Sciences, vol. 33, no. 3, pp. 471-486, 2019.

[6] J. Jose and E. I. Abdul Sathar, "Residual extropy of $k$-record values," Statistics and Probability Letters, vol. 146, pp. 1-6, 2019.

[7] J. Jose and E. I. A. Sathar, "Past extropy of $k$-records," Stochastics and Quality Control, vol. 35, no. 1, pp. 25-38, 2020.

[8] G. Qiu and K. Jia, "Extropy estimators with applications in testing uniformity," Journal of Nonparametric Statistics, vol. 30, no. 1, pp. 182-196, 2018 b.

[9] J. Yang, W. Xia, and T. Hu, "Bounds on extropy with variational distance constraint," Probability in the Engineering and Informational Sciences, vol. 33, no. 2, pp. 186-204, 2019.

[10] H. Alizadeh Noughabi and J. Jarrahiferiz, "On the estimation of extropy," Journal of Nonparametric Statistics, vol. 31, no. 1, pp. 88-99, 2019.

[11] M. Z. Raqab and G. Qiu, "On extropy properties of ranked set sampling," Statistics, vol. 53, no. 1, pp. 210-226, 2019.
[12] F. Lad, G. Sanfilippo, and G. Agro, "The duality of entropy/ extropy, and completion of the Kullback information complex," Entropy, vol. 20, no. 8, p. 593, 2018.

[13] A. S. Krishnan, S. M. Sunoj, and N. Unnikrishnan Nair, "Some reliability properties of extropy for residual and past lifetime random variables," Journal of the Korean Statistical Society, vol. 49, no. 2, pp. 457-474, 2020.

[14] S. M. A. Jahanshahi, H. Zarei, and A. H. Khammar, "On cumulative residual extropy," Probability in the Engineering and Informational Sciences, vol. 34, no. 4, pp. 605-625, 2020.

[15] E. I. Abdul Sathar and R. Dhanya Nair, "On dynamic survival extropy," Communications in Statistics-Theory and Methods, vol. 50, no. 6, pp. 1295-1313, 2021.

[16] U. Kamps, "A concept of generalized order statistics," Journal of Statistical Planning and Inference, vol. 48, no. 1, pp. 1-23, 1995.

[17] P. Pawlas and D. Szynal, "Recurrence relations for single and product moments of lower generalized order statistics from the inverse Weibull distribution," Demonstratio Mathematica, vol. 34, no. 2, pp. 353-358, 2001.

[18] M. Burkschat, U. Kamps, and E. Cramer, "Dual generalized order statistics," Metron, vol. 61, no. 1, pp. 13-26, 2003.

[19] H. A. David, M. J. O'Connell, and S. S. Yang, "Distribution and expected value of the rank of a concomitant of an order statistic," Annals of Statistics, vol. 5, pp. 216-223, 1977.

[20] D. Morgentern, "Einfache beispiele zweidimensionaler verteilunngen," Mitteilungsblatt Fur Mathemtische Statistik, vol. 8, pp. 234-235, 1956.

[21] M. I. Beg and M. Ahsanullah, "Concomitants of generalized order statistics from Farlie-Gumbel-Morgenstern distributions," Statistical Methodology, vol. 5, no. 1, pp. 1-20, 2008.

[22] M. Q. Shahbaz, M. Ahsanullah, S. H. Shahbaz, and B. M. AlZahrani, Ordered Random Variables: Theory and Applications, Atlantis Press, Dordrecht, Netherlands, 2016.

[23] M. M. Mohie El-Din, M. M. Amein, N. S. A. Ali, and M. S. Mohamed, "Residual and past entropy for concomitants of ordered random variables of Morgenstern family," Journal of Probability and Statistics, vol. 2015, Article ID 159710, 6 pages, 2015.

[24] S. Wang, "An actuarial index of the right-tail risk," North American Actuarial Journal, vol. 2, no. 2, pp. 88-101, 1998.

[25] M. Rao, Y. Chen, B. C. Vemuri, and F. Wang, "Cumulative residual entropy: a new measure of information," IEEE Transactions on Information Theory, vol. 50, no. 6, pp. 1220-1228, 2004.

[26] T. L. Lai and H. Robbins, "Maximally dependent random variables," Proceedings of the National Academy of Sciences of the United States of America, vol. 73, no. 2, pp. 286-288, 1976.

[27] A. Gravey, "A simple construction of an upper bound for the mean of the maximum of $\mathrm{n}$ identically distributed random variables," Journal of Applied Probability, vol. 22, no. 4, pp. 844-851, 1985.

[28] M. Shaked and J. G. Shanthikumar, Stochastic Orders, Springer, New York, NY, USA, 2007.

[29] E. A. Nadaraya, "On estimating regression," Theory of Probability and Its Applications, vol. 9, no. 1, pp. 141-142, 1964.

[30] R. Pyke, "Spacings," Journal of the Royal Statistical Society: Series B (Methodological), vol. 27, no. 3, pp. 395-436, 1965.

[31] D. Kasilingam, S. P. S. Prabhakaran, D. K. Rajendran, V. Rajagopal, T. S. Kumar, and A. Soundararaj, "Exploring the growth of COVID-19 cases using exponential modelling across 42 countries and predicting signs of initial containment using machine learning," Transboundary and Emerging Diseases, 2020, In press. 